\title{
DYNAMICS OF TUPLES OF MATRICES IN JORDAN FORM
}

\author{
GEORGE COSTAKIS AND IOANNIS PARISSIS
}

Abstract. A tuple $\left(T_{1}, \ldots, T_{k}\right)$ of $n \times n$ matrices over $\mathbb{R}$ is called hypercyclic if for some $x \in \mathbb{R}^{n}$ the set $\left\{T_{1}^{m_{1}} T_{2}^{m_{2}} \cdots T_{k}^{m_{k}} x: m_{1}, m_{2}, \ldots, m_{k} \in \mathbb{N}_{0}\right\}$ is dense in $\mathbb{R}^{n}$. We prove that the minimum number of $n \times n$ matrices in Jordan form over $\mathbb{R}$ which form a hypercyclic tuple is $n+1$. This answers a question of Costakis, Hadjiloucas and Manoussos.

Mathematics subject classification (2010): Primary: 47A16; Secondary: 11J72, 15A21. Keywords and phrases: Hypercyclic operator, Jordan form, Kronecker's theorem.

\section{REFERENCES}

[1] F. Bayart, É. Matheron, Dynamics of linear operators, Cambridge Tracts in Mathematics 179, 2009, xiv+337, Cambridge University Press. http://dx.doi.org/10.1017/CB09780511581113.

[2] G. Costakis, D. Hadjiloucas, A. Manoussos, Dynamics of tuples of matrices, Proc. Amer. Math. Soc. 137, 3 (2009), 1025-1034.

[3] G. Costakis, D. Hadjiloucas, A. Manoussos, On the minimal number of matrices which form a locally hypercyclic, non-hypercyclic tuple, Journal of Mathematical Analysis and Applications 365, 1 (2010), 229-237.

[4] N. S. Feldman, Hypercyclic pairs of coanalytic Toeplitz operators, Integral Equations Operator Theory 58, 2 (2007), 153-173.

[5] N. S. Feldman, Hypercyclic tuples of operators and somewhere dense orbits, J. Math. Anal. Appl. 346, 1 (2008), 82-98.

[6] N. S. Feldman, Hypercyclic Tuples of Operators, Mini-workshop: Hypercyclicity and Linear Chaos, Oberwolfach Rep. 3, 3 (2006), 2227-2276, http://dx . doi .org/10.4171/OWR/2006/37.

[7] G. H. Hardy, E. M. Wright, An introduction to the theory of numbers, Sixth Edition, xxii+621, Oxford University Press, 2008.

[8] M. JAVAHERI, Semigroups of real functions with dense orbits, 2009, http://arxiv.org/abs/0908.3316.

[9] L. KÉRCHY, Cyclic properties and stability of commuting power bounded operators, Acta Sci. Math. (Szeged) 71, 1-2 (2005), 299-312.

[10] C. KITAI, Invariant closed sets for linear operators, Univ. of Toronto, 1982.

[11] M. N. KolounTZaKIS, Unpublished Manuscript.

[12] S. G. KrantZ, Function theory of several complex variables, Reprint of the 1992 edition, xvi+564, AMS Chelsea Publishing, Providence, RI, 2001. 